\title{
ON THE ACCUMULATION OF LIMIT CYCLES
}

\author{
L. M. PERKO
}

\begin{abstract}
Limit cycles of a planar analytic system can only accumulate at a critical point or on a graphic; the critical points are isolated; and the inner and outer boundaries of any continuous band of cycles consist of either a critical point or graphic.
\end{abstract}

The main purpose of this paper is to prove that limit cycles of an analytic system

$$
\dot{x}=X(x, y), \quad \dot{y}=Y(x, y)
$$

can only accumulate at a critical point, on a cycle, or on a graphic of (1). Dulac [1] based the proof of his theorem, that polynomial systems have at most a finite number of limit cycles, on this result. And the more recent work of Chicone and Shafer [2], showing that in any bounded region of the plane a quadratic system has at most a finite number of limit cycles, is also based on this result. Even though several important papers have been based on this result, it has never been proved. The proof does follow from the Poincaré-Bendixson Theorem, as stated in [2]; however it does not follow directly since this result is concerned with the limit set of a sequence of limit cycles of (1) rather than the limit set of a trajectory of (1). A detailed proof of this important result is given in this paper.

It is well known that the analyticity of the Poincare map in the neighborhood of a cycle of (1) [3, Theorem XIII, p. 47], implies that limit cycles cannot accumulate on a cycle of (1). Dulac [1] claimed to have shown that it is also the case that limit cycles cannot accumulate at a critical point or on a graphic of (1); however, except for the case when the graphic is a single separatrix loop at a saddle point, there are difficulties in Dulac's proof (cf. [2, pp. 585 and 601]). A graphic can be described as the connected union of a finite number of compatibly oriented separatrix cycles of (1). A precise definition of a graphic is given by Francoise and Pugh in [4] and in $\S 2$ of this paper.

Two other important results are also proved in this paper. One of these results, that the critical points of a relatively prime analytical system are isolated, is well known; however, the proof does not seem to be available in the literature. This result was known at least as early as the first part of this century. In fact, Bendixson [5, p. 30], states that "one knows that each singular point is an isolated singularity"; however, Bendixson does not prove this result, nor does he cite a proof.

The other result, that the inner or outer boundary of the region covered by a continuous band of cycles of the system (1) is either a critical point or a graphic of (1) on the Poincaré sphere, is a new result. It is not surprising in view of Duff's

Received by the editors July 24, 1985 and, in revised form, February 3, 1986.

1980 Mathematics Subject Classification (1985 Revision). Primary 34C05, 34C25.

Key words and phrases. Limit cycles, Dulac's theorem.

This work was supported by a Northern Arizona University Research Grant. 
result [6], that the inner and outer boundaries of the region covered by a family of limit cycles in a family of rotated vector fields each contain a critical point.

1. On the accumulation of critical points. In this section it is shown that the critical points of a relatively prime analytic system are isolated; i.e., the analytic system (1) cannot have an infinite number of critical points in a bounded region $\Omega$ of the plane unless the functions $X$ and $Y$ have a common analytic factor in $\Omega$. The analytic functions $X(x, y)$ and $Y(x, y)$ are said to have a common analytic factor $F(x, y)$ in $\Omega$ if

$$
X(x, y)=F(x, y) G(x, y) \quad \text { and } \quad Y(x, y)=F(x, y) H(x, y),
$$

where $F, G$, and $H$ are analytic in $\Omega$.

THEOREM 1. Let $\Omega$ be a bounded region of the plane and let $X$ and $Y$ be analytic in $\Omega$. If $X$ and $Y$ have no common analytic factor in a neighborhood of any point in $\Omega$, then the system (1) has a finite number of critical points in any closed subset of $\Omega$.

ProOF. Assume that (1) has an infinite number of critical points $P_{n}$ in a closed subset $A \subset \Omega$. Then the sequence $P_{n}$ has an accumulation point in $A$. By translating the origin to that point, it may be assumed that (1) has an accumulation of critical points at the origin; i.e., that the analytic system

$$
X(x, y)=0, \quad Y(x, y)=0
$$

has an accumulation of solutions at the origin. Then by continuity, $X(0,0)=$ $Y(0,0)=0$.

If $X_{y}^{(n)}(0,0)=Y_{y}^{(n)}(0,0)=0$ for all $n$, then $X$ and $Y$ have $x$ as a common analytic factor in a neighborhood of the origin; thus, either

$$
X_{y}^{(n)}(0,0) \neq 0 \quad \text { or } \quad Y_{y}^{(n)}(0,0) \neq 0
$$

for some $n \geq 0$.

First, consider the case where

$$
\begin{aligned}
& X(0,0)=X_{y}(0,0)=\cdots=X_{y}^{(n-1)}(0,0)=0, \quad X_{y}^{(n)}(0,0) \neq 0, \\
& Y(0,0)=Y_{y}(0,0)=\cdots=Y_{y}^{(m-1)}(0,0)=0, \quad Y_{y}^{(m)}(0,0) \neq 0 .
\end{aligned}
$$

It then follows from the Weierstrass Preparation Theorem (cf. [7, Theorem 1, p. 188]) that

and

$$
X(x, y)=\left[y^{n}+a_{1}(x) y^{n-1}+\cdots+a_{n}(x)\right] A(x, y)
$$

$$
Y(x, y)=\left[y^{m}+b_{1}(x) y^{m-1}+\cdots+b_{m}(x)\right] B(x, y),
$$

where the $a_{j}(x), j=1, \ldots, n$, and $b_{j}(x), j=1, \ldots, m$, are analytic in a neighborhood of $x=0$ with $a_{j}(0)=b_{j}(0)=0$, and where $A$ and $B$ are analytic and nonvanishing in a neighborhood of the origin. Thus, the equation $X(x, y)=0$ has at most $n$ solution branches; and furthermore, according to [8, Lemma 1, p. 396], each branch $y=f(x)$ of $X(x, y)=0$ can either be expanded in powers of $x^{1 / q}$ for some integer $q$ as

$$
f(x)=\sum_{j=1}^{\infty} \alpha_{j} x^{j / q},
$$


where the series converges and represents an analytic function of $x$ at least for $0<x<\delta$, or $f(x)$ can be expanded in powers of $(-x)^{1 / q}$ for some integer $q$ as

$$
f(x)=\sum_{j=1}^{\infty} \alpha_{j}(-x)^{j / q}
$$

where the series converges and represents an analytic function of $x$ at least for $-\delta<$ $x<0$. A similar statement holds concerning the solution branches of the equation $Y(x, y)=0$. Thus, since $X(x, y)=0$ and $Y(x, y)=0$ each only have a finite number of solution branches, it follows that if the system (2) has an accumulation point of solutions at the origin, then one of the solution branches $y=f(x)$ of $X(x, y)=0$ must be equal to one of the solution branches $y=g(x)$ of $Y(x, y)=0$ at an infinite number of points $x_{j}, j=1,2,3, \ldots$, accumulating at zero.

But if

$$
f(x)=\sum_{j=1}^{\infty} \alpha_{j} x^{j / q} \text { and } g(x)=\sum_{j=1}^{\infty} \beta_{j} x^{j / p}
$$

for $0 \leq x<\delta$ and we define $\xi=x^{1 / r}$ for $0 \leq x<\delta$, where $r$ is the least common multiple of the integers $p$ and $q$, then $r / q=k$ and $r / p=i$ are integers and the functions

$$
\phi(\xi)=\sum_{j=1}^{\infty} \alpha_{j} \xi^{k j} \text { and } \psi(\xi)=\sum_{j=1}^{\infty} \beta_{j} \xi^{i j}
$$

are analytic for $|\xi|<\delta^{1 / r}$. Furthermore, $\phi(\xi)$ and $\psi(\xi)$ are equal at an infinite number of points, $\xi_{j}=x_{j}^{1 / r}, j=1,2,3, \ldots$, accumulating at zero. Thus, $\phi(\xi)=$ $\psi(\xi)$ for $|\xi|<\delta^{1 / r}$; and since $f(x)=\phi\left(x^{1 / r}\right)$ and $g(x)=\psi\left(x^{1 / r}\right)$, it follows that

$$
f(x)=g(x)
$$

for $0 \leq x<\delta$. Furthermore, $f(x)$ and $g(x)$ are analytic for $0<x<\delta$. A similar argument shows that $f(x)=g(x)$ for $-\delta<x \leq 0$ when $f(x)$ and $g(x)$ are analytic for $-\delta<x<0$.

But if $X(x, y)=0$ along the solution branch $y=f(x)$ which is analytic for $0<x<\delta$ or for $-\delta<x<0$, it follows from the corollary to Theorem 4 in [7, p. 194] that $y-f(x)$ is an analytic factor of $X(x, y)$ for $|y|<\delta$ and for $0<x<\delta$ or $-\delta<x<0$ respectively. Similarly, $Y(x, y)$ has $y-g(x)$ as an analytic factor for $|y|<\delta$ and $0<x<\delta$ or $-\delta<x<0$. But $y-f(x)=y-g(x)$ for $|y|<\delta$ and $0<x<\delta$ or $-\delta<x<0$, and this contradicts the hypothesis that $X$ and $Y$ do not have a common analytic factor in a neighborhood of any point in $\Omega$.

Finally, consider the case where $X_{y}^{(n)}(0,0)=0$ for all $n$ and $Y(0,0)=Y_{y}(0,0)=$ $\cdots=Y_{y}^{(m-1)}(0,0)=0$, but $Y_{y}^{(m)}(0,0) \neq 0$. Then $X(x, y)$ can be written as

$$
X(x, y)=x^{n}\left[a_{0}(x)+a_{1}(x) y+a_{2}(x) y^{2}+\cdots\right]=x^{n} \tilde{X}(x, y),
$$

where $n \geq 1$ is maximal. The fact that $n$ is maximal implies that not all of the $a_{j}(0)=0$ since $X$ is not identically zero. Since $X(0, y)=0$ and by the Weierstrass Preparation Theorem $Y(0, y)=y^{m} B(0, y)$, where $B(0, y) \neq 0$ for $|y|<\delta$, it follows that the origin is the only solution of $(2)$ along the $y$-axis in some neighborhood of 
the origin. If $a_{0}(0) \neq 0$, then the only branch of $X(x, y)=0$ is the $y$-axis and we are done. If $a_{0}(0)=\cdots=a_{k-1}(0)=0$ and $a_{k}(0) \neq 0$, then

$$
\tilde{X}(0,0)=\tilde{X}_{y}(0,0)=\cdots=\tilde{X}_{y}^{(k-1)}(0,0)=0, \quad \tilde{X}_{y}^{(k)}(0,0) \neq 0
$$

and we can repeat the above argument for the analytic functions $\tilde{X}(x, y)$ and $Y(x, y)$ to show that (2) cannot have an infinite number of solutions accumulating at the origin. This completes the proof of Theorem 1.

2. On the accumulation of limit cycles. In this section it is shown that limit cycles of a relatively prime analytic system (1) can accumulate only at a critical point, on a cycle, or on a graphic of (1). This is equivalent to showing that the limit set of any bounded sequence of limit cycles of (1) is either a critical point, a cycle, or a graphic of (1). The limit set $S=\lim _{n \rightarrow \infty} L_{n}$ of a sequence of limit cycles $L_{n}$ of (1) is defined as the set

$$
S=\left\{P \in \mathbf{R}^{2} \mid \exists \text { a sequence of points } P_{n} \in L_{n} \text { with } P_{n} \rightarrow P \text { as } n \rightarrow \infty\right\} \text {. }
$$

Since trajectories of (1) do not cross, it follows that any sequence of limit cycles of (1) consists of a countable number of nested sequences of limit cycles of (1). A sequence of limit cycles is said to be nested if for all $i$ and $j$ either $L_{i} \subset \operatorname{Int} L_{j}$ or $L_{j} \subset$ Int $L_{i}$. A sequence of limit cycles is called monotone if it is either expanding (i.e., $L_{n} \subset$ Int $L_{n+1}$ ) or contracting (i.e., $L_{n+1} \subset \operatorname{Int} L_{n}$ ). For a monotone sequence of limit cycles $L_{n}$, the limit set is equal to the boundary of the set

$$
\bigcup_{n=1}^{\infty} \operatorname{Int} L_{n} \quad \text { or } \bigcap_{n=1}^{\infty} \operatorname{Int} L_{n}
$$

when $L_{n}$ is an expanding or contracting sequence of limit cycles respectively. Furthermore, it can be shown that any point $P \in \lim _{n \rightarrow \infty} L_{n}$ belongs to the limit set of a monotone subsequence of $L_{n}$. In Theorem 2 below it is shown that the limit set of any bounded monotone sequence of limit cycles of $(1)$ is either a critical point or a graphic of (1). Since any set $S$ is the union of its connected components, it follows that the limit set of any sequence of limit cycles of (1) is a union of critical points and graphics of (1); i.e., it follows from Theorem 2 below that limit cycles of (1) can only accumulate at a critical point or on a graphic of (1).

As was stated in the introduction, a graphic of (1) can be described as the connected union of a finite number of compatibly oriented separatrix cycles of (1). More precisely, as in Franciose and Pugh [4, pp. 4 and 5] a graphic is a loop formed by singular points and normally oriented separatrices connecting them:

$$
P_{1}, \Gamma_{1}, P_{2}, \Gamma_{2}, \ldots, P_{m}, \Gamma_{m}, P_{m+1}=P_{1}
$$

such that

(1) for $j=1, \ldots, m, \alpha\left(\Gamma_{j}\right)=P_{j}$, where $\alpha\left(\Gamma_{j}\right)$ is the $\alpha$-limit set of $\Gamma_{j}$, and $\omega\left(\Gamma_{j}\right)=P_{j+1}$, where $\omega\left(\Gamma_{j}\right)$ is the $\omega$-limit set of $\Gamma_{j}$, and

(2) for $j=1, \ldots, m-1$, normal orientations $n_{j}$ of separatrices are compatible in the sense that if $\Gamma_{j}$ has left-hand orientation, then so does $\Gamma_{j+1}$ (cf. Figure 1).

The second condition above is equivalent to requiring that each simple loop or separatrix cycle contained in the graphic has the same orientation as some cycle does with respect to a given point $P_{0}$. For example, Figure 2 shows several graphics in which each separatrix cycle is positively oriented with respect to the point $P_{0}$. The 


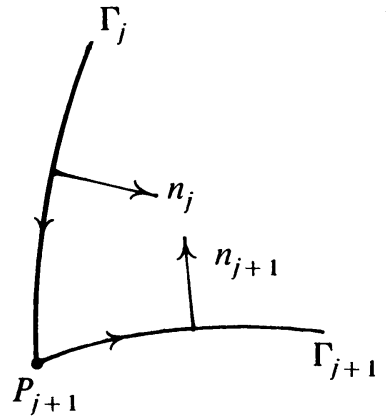

(a)

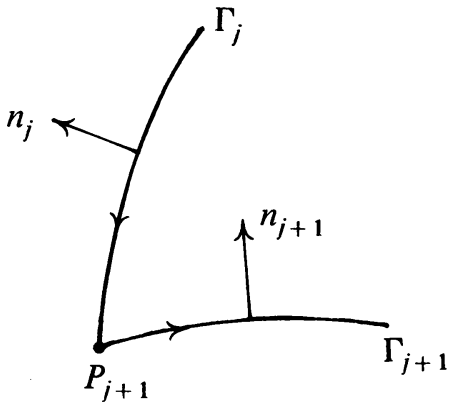

(b)

FIGURE 1. The separatrices are compatibly oriented in (a) but not in (b)

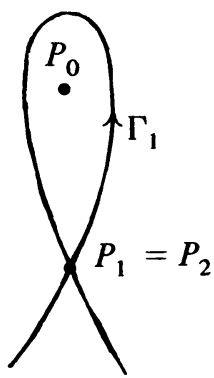

(a)

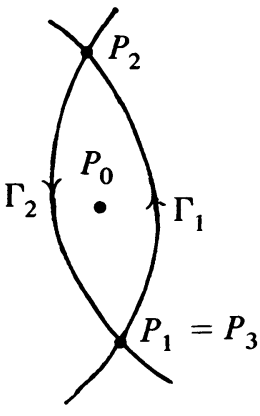

(b)

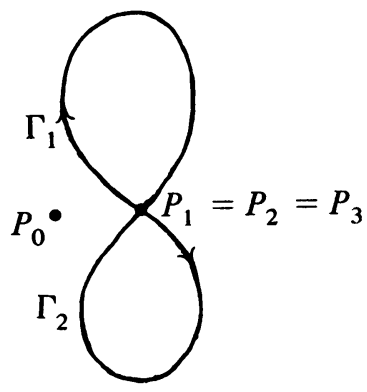

(c)

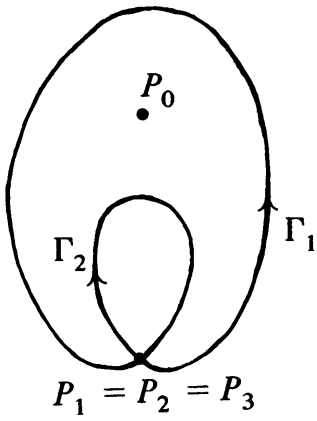

(d)

FIGURE 2. Graphics

graphic in Figure 2(a) occurs in the quadratic system shown in [9, pp. 156-157] and the graphic in Figure 2(c) occurs in the cubic system in [8, p. 11]. The concept of a graphic allows a succinct statement of the Poincaré-Bendixson Theorem: The $\alpha$ - or $\omega$-limit set of any trajectory of the relatively prime analytic system (1), contained in a bounded region of the plane, is either a critical point, a cycle, or a graphic of (1).

With these definitions in mind, we now state the main result of this paper. It is assumed that the system (1) is analytic in all of $\mathbf{R}^{2}$ although this condition can be weakened. Also, the analyticity of the Poincaré map implies that the limit set $S$ cannot be a cycle and this is incorporated in the statement of the following theorem.

THEOREM 2. If $L_{n}$ is a monotone sequence of limit cycles of the relatively prime analytic system (1), contained in a bounded region of the plane, then the limit set $S=\lim _{n \rightarrow \infty} L_{n}$ is either a critical point or a graphic of (1) if $L_{n}$ is a contracting sequence, and $S$ is a graphic of (1) if $L_{n}$ is an expanding sequence.

This theorem is proved by the following sequence of lemmas. The hypotheses of Theorem 2 are assumed to hold in stating these lemmas. The proof of the first 
lemma is essentially the same as the proof of Theorem 1.1 on p. 390 in [10]. In the remainder of this paper, $\Gamma_{p}$ will denote the trajectory of $(1)$ through the point $P$ at $t=0 ; \Gamma(t, P)$ will denote the point reached at time $t$ on the trajectory $\Gamma_{p}$; and $\alpha\left(\Gamma_{p}\right)$ and $\omega\left(\Gamma_{p}\right)$ will denote the $\alpha$ - and $\omega$-limit sets of the trajectory $\Gamma_{p}$ respectively.

LEMMA 2.1. $S$ is a nonempty, closed, connected set.

LEMMA 2.2. If $P \in S$, then $\Gamma_{p} \subset S$.

Proof. If $P \in S$ is a critical point of (1), then $\Gamma_{p}=\{P\} \subset S$. Otherwise, it follows from the continuity of solutions with respect to initial conditions that if $P_{n} \rightarrow P$ as $n \rightarrow \infty$, then for fixed $t$

$$
\lim _{n \rightarrow \infty} \Gamma\left(t, P_{n}\right)=\Gamma(t, P) .
$$

But if $P_{n} \in L_{n}$, then for each $t, \Gamma\left(t, P_{n}\right) \in L_{n}$ since $L_{n}$ is a cycle. It then follows from the definition of $S$ that for each $t, \Gamma(t, P) \in S$; i.e., $\Gamma_{p} \subset S$.

Since Lemma 2.2 implies that $S$ is an invariant set, the next lemma follows from [11, part (b) of the proposition on p. 240].

LEMMA 2.3. If $P \in S$, then $\omega\left(\Gamma_{p}\right) \subset S$ and $\alpha\left(\Gamma_{p}\right) \subset S$.

LEMMA 2.4. If for a given $P \in S, \omega\left(\Gamma_{p}\right)$ is a cycle, it follows that $\omega\left(\Gamma_{p}\right)=$ $S=\Gamma_{p}$.

Proof. By Lemma 2.3, for $P \in S, \omega\left(\Gamma_{p}\right) \subset S$. It remains to show that if $\omega\left(\Gamma_{p}\right)$ is a cycle, then $\omega\left(\Gamma_{p}\right) \supset S$. In order to show this, it is necessary to first establish that the periods of the limit cycles $L_{k}$ are uniformly bounded.

Let $Q \in \omega\left(\Gamma_{p}\right)$. Then, by Lemma 2.3, $Q \in S$. Thus, there exists a sequence $Q_{k} \in L_{k}$ such that $Q_{k} \rightarrow Q$ as $k \rightarrow \infty$. If $\omega\left(\Gamma_{p}\right)$ is a cycle of period $T$, then for $Q \in \omega\left(\Gamma_{p}\right), \Gamma(T, Q)=Q$ and it follows from continuity with respect to initial conditions that the points $\tilde{Q}_{k}=\Gamma\left(T, Q_{k}\right)$ approach $Q$ as $k \rightarrow \infty$. For $k$ sufficiently large, the points $Q_{k}$ may be chosen as the points where a transversal $\sigma$ through the noncritical point $Q$ intersects the limit cycles $L_{k}$. Then since $\Gamma(0, Q)=Q$, it follows from [11, proposition on p. 243] that in a sufficiently small neighborhood $N$ of the point $Q$, there exists a $C^{\mathbf{1}}$-function $\tau: N \rightarrow \mathbf{R}$ such that $\tau(Q)=0$ and for all $\tilde{Q} \in N, \Gamma(\tau(\tilde{Q}), \tilde{Q}) \in \sigma$. Then since $Q$ is not a critical point of (1), it follows by constructing a flow box in a sufficiently small neighborhood of $Q$, as in [11, pp. 242-243], that for the planar flow (1), the limit cycle $L_{k}$ only crosses the transversal $\sigma$ once; i.e., for sufficiently large $k$

$$
\Gamma\left(\tau\left(\tilde{Q}_{k}\right), \tilde{Q}_{k}\right)=Q_{k}
$$

i.e., $T+\tau\left(\tilde{Q}_{k}\right)$ is the period of $L_{k}$. It then follows from the continuity of $\tau$ that $\tau\left(\tilde{Q}_{k}\right) \rightarrow \tau(Q)=0$ as $k \rightarrow \infty$. And this implies that for $k$ sufficiently large, the periods of the limit cycles $L_{k}$ are uniformly bounded by $T+1$.

Now, for any point $P^{\prime} \in S$, there exists a sequence $P_{k}^{\prime} \in L_{k}$ such that $P_{k}^{\prime} \rightarrow P^{\prime}$ as $k \rightarrow \infty$; and it follows from above that if $\omega\left(\Gamma_{p}\right)$ is a cycle, then for a given sequence $Q_{k} \in L_{k}$ with $Q_{k} \rightarrow Q \in \omega\left(\Gamma_{p}\right)$ as $k \rightarrow \infty$, there exists a bounded sequence $t_{k} \in[0, T+1]$ such that $P_{k}^{\prime}=\Gamma\left(t_{k}, Q_{k}\right)$. Then by continuity of solutions with respect to initial conditions uniformly with respect to $t$ on the fixed time interval 
$[0, T+1](\mathrm{cf} .[10$, Theorem 4.3, p. 59]), it follows that for a given $\varepsilon>0$ there exists an $N$ such that for all $k \geq N$

$$
\left|P_{k}^{\prime}-\Gamma\left(t_{k}, Q\right)\right|<\varepsilon .
$$

Thus,

$$
\lim _{k \rightarrow \infty} \Gamma\left(t_{k}, Q\right)=P^{\prime}
$$

Since $\Gamma\left(t_{k}, Q\right) \in \omega\left(\Gamma_{p}\right)$ when $Q \in \omega\left(\Gamma_{p}\right)$ and $\omega\left(\Gamma_{p}\right)$ is a cycle, it follows from the fact that $\omega\left(\Gamma_{p}\right)$ is a closed set (cf. $\left[\mathbf{1 0}\right.$, p. 390]) that $P^{\prime} \in \omega\left(\Gamma_{p}\right)$. Hence, since any point $P^{\prime} \in S$ belongs to $\omega\left(\Gamma_{p}\right)$, it follows that $S \subset \omega\left(\Gamma_{p}\right)$. Thus, $S=\omega\left(\Gamma_{p}\right)$.

Finally, it follows from Lemma 2.3 and the above result that $\Gamma_{p} \subset S=\omega\left(\Gamma_{p}\right)$. And therefore by $\left[10\right.$, Lemmas 2.3 and 2.4, p. 393], it follows that $\Gamma_{p}=\omega\left(\Gamma_{p}\right)$ if $\omega\left(\Gamma_{p}\right)$ is a cycle. This completes the proof of Lemma 2.4 .

Corollary. For $P \in S, \omega\left(\Gamma_{p}\right)$ is not a cycle of $(1)$.

PRoOF. If $\omega\left(\Gamma_{p}\right)$ is a cycle, then by Lemma 2.4, $S=\omega\left(\Gamma_{p}\right)$ and the limit set $S$ is a cycle. But then it would follow from the analyticity of the Poincaré return map, $h(r)$, along a transversal to the cycle $S$ (cf., e.g., [12, Lemma 23, p. 74]) that the displacement function $d(r)=h(r)-r$ is identically equal to zero for $|r|<\delta$. This would imply that $S$ is contained in a continuous band of cycles of (1), contradicting the fact that $L_{n}$ is a sequence of limit cycles of (1) which approach $S$ as $n \rightarrow \infty$. Thus, $\omega\left(\Gamma_{p}\right)$ is not a cycle of $(1)$.

LEMMA 2.5. S contains at least one and at most a finite number of critical points of (1).

PROOF. If the sequence of limit cycles $L_{n}$ is contained in the bounded set $\Omega$, then the limit set $S \subset \bar{\Omega}$. It follows from Lemma 2.3 that for $P \in S, \omega\left(\Gamma_{p}\right) \subset \bar{\Omega}$. Thus, according to the Poincaré-Bendixson Theorem, $\omega\left(\Gamma_{p}\right)$ either contains a critical point or is a cycle of (1). However, according to the above corollary, $\omega\left(\Gamma_{p}\right)$ is not a cycle of (1). Thus $\omega\left(\Gamma_{p}\right)$ contains a critical point of (1). Since, by Lemma $2.3, \omega\left(\Gamma_{p}\right) \subset S$, it follows that $S$ contains at least one critical point of (1). By Theorem 1, the relatively prime analytic system (1) has at most a finite number of critical points in $\bar{\Omega}$. Since $S \subset \bar{\Omega}, S$ contains at most a finite number of critical points of (1).

LEMMA 2.6. $S$ is either a single critical point of (1) or $S$ is a connected set composed of a finite number of distinct critical points of (1), $P_{i}, i=1, \ldots, n$, and a finite number of distinct separatrices of $(1), \Gamma_{i}, i=1, \ldots, m$, with $m \geq n$ such that for each $i=1, \ldots, m$, there are integers $j, k \in\{1, \ldots, n\}$ such that $\omega\left(\Gamma_{i}\right)=P_{j}$ and $\alpha\left(\Gamma_{i}\right)=P_{k} ;$ and such that for each $i=1, \ldots, n$, there are integers $j, k \in\{1, \ldots, m\}$ such that $P_{i}=\omega\left(\Gamma_{j}\right)$ and $P_{i}=\alpha\left(\Gamma_{k}\right)$; furthermore, there is an integer $N$ such that for $n \geq N$, all of the separatrix cycles contained in $S$ have the same orientation with respect to some point $P_{0}$ as does the limit cycle $L_{n}$.

Proof. Given $P \in S$. If $P$ is not a critical point of (1), then, as in the proof of Lemma 2.5, $\omega\left(\Gamma_{p}\right)$ contains a critical point of (1). By the Poincaré-Bendixson Theorem, $\omega\left(\Gamma_{p}\right)$ is either a single critical point or a graphic of (1).

It is first shown that $\omega\left(\Gamma_{p}\right)$ is not a graphic of $(1)$. Suppose it is. Then by $[\mathbf{1 0}$, Theorem 3.2, p. 396], $\Gamma_{p}$ spirals toward $\omega\left(\Gamma_{p}\right)$ as $t \rightarrow \infty$, either in the exterior of $\omega\left(\Gamma_{p}\right)$ or in one of the interior components of $\omega\left(\Gamma_{p}\right)$. Suppose that $\Gamma_{p}$ is in 
the exterior of $\omega\left(\Gamma_{p}\right)$ and that $L_{n}$ is a contracting sequence of limit cycles of (1). Then since $\Gamma_{p}$ spirals toward $\omega\left(\Gamma_{p}\right)$ as $t \rightarrow \infty$, there exists a Bendixson curve, $\gamma$, composed of a segment of $\Gamma_{p}$ and a transversal to $\Gamma_{p}$ such that $\omega\left(\Gamma_{p}\right)$ lies in the interior of $\gamma$ and $\omega\left(\Gamma_{p}\right)$ is a positive distance from $\gamma$ (cf. [10, Figure 19, p. 396]). Since the limit cycles $L_{n}$ of (1) do not cross the Bendixson curve $\gamma$, either (a) all of the limit cycles $L_{n}$ are in the exterior of $\gamma$ or (b) there exists a limit cycle $L_{n}$ in the interior of $\gamma$ and in the exterior of $\omega\left(\Gamma_{p}\right)$. Since $\omega\left(\Gamma_{p}\right) \subset S$ by Lemma 2.3, it follows that for each point $P^{\prime} \in \omega\left(\Gamma_{p}\right)$ there is a sequence of points $P_{n}^{\prime} \in L_{n}$ such that $P_{n}^{\prime} \rightarrow P^{\prime}$ as $n \rightarrow \infty$; it follows that case (a) cannot occur. Since trajectories do not cross and $\Gamma_{p}$ spirals toward $\omega\left(\Gamma_{p}\right)$ as $t \rightarrow \infty$, case (b) cannot occur. It follows that $\omega\left(\Gamma_{p}\right)$ is not a graphic. A similar proof by contradiction shows that $\omega\left(\Gamma_{p}\right)$ is not a graphic when $L_{n}$ is an expanding sequence of limit cycles or when $\Gamma_{p}$ is in one of the interior components of $\omega\left(\Gamma_{p}\right)$. Thus, $\omega\left(\Gamma_{p}\right)$ consists of a single critical point of (1) and similarly it can be shown that $\alpha\left(\Gamma_{p}\right)$ consists of a single critical point of (1).

Next, assume that $S$ is not a single critical point of (1). Then, since $S$ is connected by Lemma 2.1, it follows that each critical point $P \in S$ is either the $\alpha$ or the $\omega$-limit set of some trajectory, which by Lemma 2.2 is entirely contained in $S$. Furthermore, it follows from the definition of the limit set $S$ and the continuity of solutions with respect to initial conditions that each critical point $P \in S$ is both the $\alpha$ - and the $\omega$-limit set of trajectories in $S$ and that there is a hyperbolic sector at $P$; i.e., each trajectory in $S$ is a separatrix. But, by [5, Theorem IX, p. 32], there are at most a finite number of separatrices at each critical point of (1). And since by Theorem 1 above, there are at most a finite number of critical points of (1), it follows that there are at most a finite number of separatrices in $S$; i.e., $S$ is composed of a finite number of distinct critical points, $P_{i}, i=1, \ldots, n$, and a finite number of distinct separatrices, $\Gamma_{i}, i=1, \ldots, m$, of $(1)$ as described in the statement of this lemma. Clearly, since each critical point in $S$ is the $\alpha$-limit set of some separatrix in $S$, and since a separatrix in $S$ cannot have two different critical points in $S$ as its $\alpha$-limit set, it follows that $m \geq n$.

Finally, if $S$ is not a single critical point of (1), it follows from the definition of the limit set $S$ and the continuity of solutions with respect to initial conditions that there is an integer $N$ such that for $n \geq N$ each separatrix cycle contained in $S$ has the same orientation as $L_{n}$ with respect to any point $P_{0} \in \operatorname{Int} L_{1}$ if $L_{n}$ is an expanding sequence of limit cycles, or with respect to any point $P_{0} \in \operatorname{Ext} L_{1}$ if $L_{n}$ is a contracting sequence of limit cycles. The fact that all of the limit cycles $L_{n}$ of the analytic system (1) have the same orientation for $n$ sufficiently large is established in [13]. This completes the proof of Lemma 2.6.

Theorem 2 then follows from Lemma 2.6 and the definition of a graphic since from Lemma 2.6, the definition of the limit set $S$, and the continuity of solutions with respect to initial conditions, it follows that if $S$ is not a single critical point then $S$ is a loop (i.e. a closed curve) formed by a finite number of critical points and separatrices connecting them:

$$
P_{1}, \Gamma_{1}, P_{2}, \ldots, P_{m}, \Gamma_{m}, P_{m+1}=P_{1}
$$

such that (with an appropriate renumbering of the critical points and separatrices 
in Lemma 2.6)

$$
\begin{aligned}
& P_{1}=\alpha\left(\Gamma_{1}\right), \omega\left(\Gamma_{1}\right)=P_{2}=\alpha\left(\Gamma_{2}\right), \ldots, \\
& \qquad \omega\left(\Gamma_{m-1}\right)=P_{m}=\alpha\left(\Gamma_{m}\right), \omega\left(\Gamma_{m}\right)=P_{m+1}=P_{1} ;
\end{aligned}
$$

also, the fact that the normal orientations of the separatrices are compatible follows from the last statement in Lemma 2.6.

If the sequence of limit cycles $L_{n}$ is not bounded, then it can be shown, using the above techniques, that the limit set $S$ is either a cycle on the equator of the Poincare sphere or a graphic with at least one critical point on the equator of the Poincaré sphere (cf. [9, pp. 148-149] for some examples of quadratic systems having graphics with some of their critical points and separatrices on the equator of the Poincaré sphere).

As was mentioned in the introduction, Dulac $[\mathbf{1}]$ claimed to have shown that limit cycles of analytic systems do not accumulate at critical points or on graphics. In view of Theorem 2, this would imply that analytic systems can only have a finite number of limit cycles in any bounded region of the plane. However, Dulac [1] assumed that his logarithmic series for the Poincaré return map converged in a neighborhood of a graphic; this is not true in general. Hence, the result that limit cycles of analytic systems do not accumulate at critical points or on graphics still remains to be proved. Dulac's series for the Poincaré map does converge in the neighborhood of a single separatrix loop at a saddle point, such as the one shown in Figure 2(a) above, and this implies that an infinite number of limit cycles cannot accumulate on such a graphic (cf. [2, Theorem 4.6, p. 601]). Chicone and Shafer [2] use this result to show that quadratic systems can have at most a finite number of limit cycles in any bounded region of the plane. Also, Il'yashenko [14] has recently shown, by an approach different from Dulac's, that limit cycles do not accumulate on a graphic having only hyperbolic saddle points. Several results indicating that limit cycles cannot accumulate at a critical point of (1), i.e., that (1) does not have a "center-focus", have been established in [8] and [13] by methods different from those of Dulac [1]. For example, it is well known that if $X$ or $Y$ begin with linear terms, then (1) does not have a center-focus at the origin (cf. [8]), and if the minimum degree of $X$ and $Y$ is even, then (1) does not have a center-focus at the origin (cf. [13]). Also, if $X$ and $Y$ are fourth degree polynomials, (1) does not have a center-focus (cf. $[\mathbf{1 3}]$ ).

It is conjectured that Dulac's Theorem is true; i.e., that polynomial systems have at most a finite number of limit cycles on the Poincaré sphere and that analytic systems have at most a finite number of limit cycles in any bounded region of the plane. However, these results still need to be proved and Theorem 2 above is the natural starting point for such a proof.

3. On the boundary of a continuous band of cycles. In this section it is shown that the inner and outer boundaries of a continuous band of cycles of (1) consist of either a critical point or a graphic of (1).

It is assumed that (1) has a continuous band of cycles that cover a region $R$ in the plane; i.e., for each point $P \in R, \Gamma_{p}$ is a cycle of (1). It follows that $R$ can be represented as a union of cycles:

$$
R=\bigcup_{\alpha \in(0,1)} L_{\alpha},
$$


where for each $\alpha \in(0,1), L_{\alpha}$ is a cycle of (1). The boundary, $\dot{R}$, of the region $R$ is defined as the set

$$
\begin{aligned}
& \dot{R}=\left\{P \in \mathbf{R}^{2} \mid \exists \text { a sequence } \alpha_{n} \in(0,1)\right. \text { and a sequence of points } \\
& P_{n} \in L_{\alpha_{n}} \text { with } P_{n} \rightarrow P \text { as } n \rightarrow \infty \text {; and such that for all } \\
& \left.\varepsilon>0 \exists Q \in N_{\varepsilon}(P) \text { such that } \Gamma_{Q} \text { is not a cycle of }(1)\right\} \text {. }
\end{aligned}
$$

As in $\S 2, \Gamma_{p}$ denotes the trajectory of (1) through the point $P$ at $t=0$, and $N_{\varepsilon}(P)$ denotes an $\varepsilon$-neighborhood of the point $P \in \mathbf{R}^{2}$.

If $R$ is a continuous band of cycles of $(1)$, contained in a bounded region of the plane, it can be shown that $R$ is homeomorphic to an open annulus. The boundary, $\dot{R}$, of $R$ will therefore have two components called the inner and outer boundaries of $R$. It is assumed that the system (1) is analytic in all of $\mathbf{R}^{2}$ although this condition can be weakened.

THEOREM 3. If $R$ is a continuous band of cycles of the relatively prime analytic system (1), contained in a bounded region of the plane, then the inner boundary of $R$ is either a single critical point or a graphic of (1) and the outer boundary of $R$ is a graphic of (1).

Before proving this theorem, some examples illustrating the theorem and its extension to the Poincare sphere are cited. It was shown in $[9, \mathrm{p} .158]$, that the quadratic system

$$
\dot{x}=y+y^{2}, \quad \dot{y}=-2 x-x y
$$

has a continuous band of cycles $R$, the inner boundary consisting of the critical point at the origin and the outer boundary consisting of a single separatrix loop at the saddle point $(0,-1)$ (cf. Figure $2(\mathrm{a})$ above). It can be shown that if $R$ is not contained in a bounded region of the plane, then the outer boundary of $R$ either consists of a cycle on the equator of the Poincare sphere or it consists of a graphic with at least one critical point on the equator of the Poincare sphere (cf. [9, Figure 8 on p. 156] for an example of this latter case). It was shown in [8, p. 211] that the cubic system

$$
\dot{x}=y+y\left(x^{2}+y^{2}\right), \quad \dot{y}=x-x\left(x^{2}+y^{2}\right)
$$

has a continuous band of cycles $R$, the inner boundary consisting of a graphic which is composed of two separatrix loops at the saddle at the origin (cf. Figure 2(c) above). The outer boundary of this continuous band of cycles is a cycle on the equator of the Poincaré sphere.

Theorem 3 can be proved by establishing the same sequence of lemmas used in proving Theorem 2 in $\S 2$ with $S$ denoting either the inner or outer boundary of $R$ in those lemmas; the proofs in $\S 2$ require only minor modifications. However, the referee in reviewing this paper pointed out a method for obtaining Theorem 3 directly from the Poincaré-Bendixson Theorem. The method consists of modifying the vector field $W$, defined by (1), in order to obtain a vector field $Z$ having a trajectory whose $\omega$-limit set is the inner or outer boundary $S$ of the region $R$ in Theorem 3.

In order to illustrate this method for proving Theorem 3, consider the case when $S$ is the outer boundary of the region $R$ and the cycles $L_{\alpha}$ composing $R$ are negatively oriented. In this case, the vector field $Z$ is constructed as follows: For a 
noncritical point $P \in S$, let $N=[-1,1] \times[-1,1]$ and, as $\left[\mathbf{1 1}\right.$, p. 242], let $\Psi: N \rightarrow \mathbf{R}^{2}$ be a flow box centered at $P=\Psi(0,0)$ with the parallel line segments below the $x$-axis in $N$ corresponding to the cycles $L_{\alpha}$ near $P$ in $\Psi(N)$. Let $f: N \rightarrow[0,1]$ be a smooth function with $f=0$ outside $N / 2=\left[-\frac{1}{2}, \frac{1}{2}\right] \times\left[-\frac{1}{2}, \frac{1}{2}\right]$ and $f=1$ inside $N / 4$. The vector field $Z$ is then defined to be the vector field $W$ outside of $\Psi(N / 2)$ and inside of $\Psi(N / 2)$ it is defined to be the image under $\Psi$ of the vector field in $N$ defined by $\dot{x}=1, \dot{y}=-f(x, y) y$. Observe that the trajectory through $P$ is the same for $W$ and $Z$ and that $P$ is in the $\omega$-limit set of any $Z$-trajectory $\Gamma_{Q}$ with $Q \in \Psi(N / 8)$. Also, observe that inside $\Psi(N / 2)$ each $Z$-trajectory crosses each cycle $L_{\alpha}$ from its interior to its exterior. Thus, any $Z$-trajectory which enters $\Psi(N / 2)$ on a cycle $L_{\alpha}$, leaves $\Psi(N / 2)$ on a cycle $L_{\beta}$ with $\beta>\alpha$ (where $L_{\alpha} \subset \operatorname{Int} L_{\beta}$ ). It follows that for $Q \in \Psi(N / 8), S=\omega\left(\Gamma_{Q}\right)$. Thus, it follows from the Poincaré-Bendixson Theorem applied to the vector field $Z$ (which has only a finite number of critical points) that $S$ is a critical point, a cycle, or a graphic of $Z$ and hence, also of $W$. But $S$ cannot be a cycle since, by the analyticity of the Poincaré map in a neighborhood of a cycle, $S$ would then belong to a continuous band of cycles which would contradict the definition of the boundary of the region $R$; and clearly if $S$ is the outer boundary of the region $R$, it cannot consist of a single critical point. This proves Theorem 3 in the above case. The remaining cases can be treated in a similar manner.

The fact that Theorem 3 follows directly from the Poincaré-Bendixson Theorem, as illustrated above, brings up an interesting question: Does Theorem 2 follow directly from the Poincaré-Bendixson Theorem; i.e., can the vector field $W$, defined by (1), be modified to obtain a vector field $Z$ having the limit set $S$ in Theorem 2 as the $\omega$-limit set of one of its trajectories? It is natural to try the above approach used to prove Theorem 3 with the vector field defined in $N$ by $\dot{x}=1, \dot{y}=-k f(x, y) y$, where $k$ is a suitably large constant. However, it is not apparent that this approach will work for any $k>0$ since, under the hypotheses of Theorem 2 , there are trajectories arbitrarily close to any point in $S$ which spiral away from $S$. Perhaps another modification of $W$ will work. This problem of constructing such a modification of the vector field $W$ is left as an interesting open problem in this paper.

The following related question was also posed by the referee: Is there a graphic of some vector field $W$ which is not the $\omega$-limit set of a trajectory of some vector field $Z$ ? If Dulac's Theorem is valid, the answer is no; i.e., any graphic of an analytic vector field $W$ can only exist as the limit set of a trajectory of $W$, the limit set of a sequence of limit cycles of $W$, or the boundary of a continuous band of cycles of $W$. In the first case, the answer to the above question is clearly no, the second case is ruled out by Dulac's Theorem, and in the third case the answer is clearly no in view of the above proof of Theorem 3 .

This completes this paper on the accumulation of critical points, limit cycles, and cycles of analytic systems. The author would like to take this opportunity to express his sincere appreciation to the referee for his careful reading of the first version of this paper and for pointing out the above method for proving Theorem 3 and the interesting questions that this raises.

\section{REFERENCES}

1. H. Dulac, Sur les cycles limites, Bull. Soc. Math. France 51 (1923), 45-188.

2. C. Chicone and D. S. Shafer, Separatrix and iimit cycles of quadratic systems and Dulac's theorem, Trans. Amer. Math. Soc. 278 (1983), 585-612. 
3. H. Poincaré, Mémoire sur les courbes définies par une equation différentielle, J. Mathématiques 7 (1881), 375-422; Oeuvre (1880-1890), Gauthier-Villar, Paris, pp. 1-221.

4. J. P. Francoise and C. C. Pugh, Keeping track of limit cycles, Berkeley Report, PAM-239, 1984.

5. I. Bendixson, Sur les courbes définies par des équations différentielles, Acta Math. 24 (1901), 1-88.

6. G. F. D. Duff, Limit cycles and rotated vector fields, Ann. of Math. (2) 67 (1953), 15-31.

7. S. Bochner and W. T. Martin, Several complex variables, Princeton Univ. Press, Princeton, N.J., 1948.

8. A. A. Andronov et al., Theory of bifurcations of dynamical systems on a plane, Kefer Press, Jerusalem, 1971.

9. L. M. Perko and Shu Shih-Lung, Existence, uniqueness, and nonexistence of limit cycles for a class of quadratic systems in the plane, J. Differential Equations 53 (1984), 146-171.

10. E. A. Coddington and N. Levinson, Theory of ordinary differential equations, McGrawHill, New York, 1955.

11. M. W. Hirsch and S. Smale, Differential equations, dynamical systems, and linear algebra, Academic Press, New York, 1974.

12. A. A. Andronov et al., Qualitative theory of second order dynamical systems, Wiley, New York, 1973.

13. L. M. Perko, Center-foci for analytic systems (in preparation).

14. Y. Il'yashenko, Limit cycles of polynomial vector fields, Functional Anal. Appl. 18 (1984), 199-209.

DePartment of Mathematics, Northern ARIzona University, FlagstafF, ARIZONA 86011 